\title{
Changes in Quality Characteristics of Pork Patties Containing Multilayered Fish Oil Emulsion during Refrigerated Storage
}

\author{
Yeon-Ji Jo', Yun-Joong Kwon², Sang-Gi Min ${ }^{1}$, and Mi-Jung Choi* \\ Department of Bioresources and Food Science, Konkuk University, Seoul 143-701, Korea \\ ${ }^{1}$ Department of Bioindustrial Technologies, Konkuk University, Seoul 143-701, Korea \\ ${ }^{2}$ Department of Food Science and Biotechnology, Kyonggi University, Suwon 443-760, Korea
}

\begin{abstract}
This study was performed to determine the effect of multilayered fish oil (FO) emulsion without or with trans-cinnamaldehyde on pork patties. Multilayered FO (-primary, -secondary, -tertiary) emulsions were prepared using a layer-by-layer deposition technique with Tween 20, chitosan, and low methoxyl pectin, and were added to pork patties at the same concentration. Pork patties were then stored for $20 \mathrm{~d}$ in a refrigerator $\left(5^{\circ} \mathrm{C}\right)$ to study changes in quality. The results showed that the $\mathrm{pH}$ value of all samples significantly decreased but cooking loss increased during storage $(p<0.05)$. However, water-holding capacity and moisture content showed no remarkable difference between treatments and storage periods $(p>0.05)$. All pork patties containing multilayered FO (treated samples) showed higher values for lightness and significantly lower values for yellowness compared to control pork patties (untreated sample). Lipid oxidation was higher in treated pork patties than in control pork patties during storage. In addition, lipid oxidation and total viable bacterial count in pork patties decreased as the number of coating layers increased. However, hardness, cohesiveness, and springiness of all samples showed no significant change during storage $(p>0.05)$ as compared to fresh pork patties. Furthermore, these did not remarkable change with addition of trans-cinnamaldehyde in all pork patties. From our results, we suggest that FO emulsion did not affect the texture characteristics of fresh pork patties, indicating that it could be used to improve the quality of pork patties by contributing high-quality fat such as unsaturated fatty acids.
\end{abstract}

Key words: fish oil, multilayered emulsions, lipid oxidation, texture, pork patties

\section{Introduction}

Emulsion-type meat products are commercially produced from poultry, pork, or beef emulsified with back fat. Although meat products are an important source of protein, vitamins, and minerals, they contain large quantities of fat, saturated fatty acids, salt, and cholesterol (Jiménez-Colmenero et al., 2001). High fat intake causes hypertension, obesity, cardiovascular disease, and coronary heart disease (Colmenero, 1996). Many studies have reported that high intake of fat is related to risk of obesity and cardiovascular disease resulting from low-density lipoprotein-cholesterol (LDL-cholesterol) production. Therefore, several attempts are being made to reduce animal fat contents in meat products.

Josquin et al. (2012) replaced animal fat in emulsified

\footnotetext{
*Corresponding author: Mi-Jung Choi, Department of Bioresources and Food Science, Konkuk University, Seoul 143-701, Korea. Tel: +82-2-450-3048, Fax: +82-2-450-3726, E-mail: choimj@konkuk.ac.kr
}

sausages with olive to enhance the polyunsaturated fatty acid (PUFA) content. They also attempted to increase PUFA content by adding linseed oil, rapeseed oil, and fish oil to meat products. Therefore, reduction of fat and addition of high-quality fatty acid in meat products is desirable as they contain fewer calories. This positive effect could be further enhanced by substituting animal fat (Lurueña-Martýìnez et al., 2004).

In general, it is reported that the addition of fish oil (FO) in meat product formulations prevents and modulates diseases such as coronary heart disease, cancer, hypertension, and arthritis (Shahidi and Miraliakbari, 2004; Uauy and Valenzuela, 2000). However, problems associated with addition of FO to meat products include a strong fishy flavor and oxidative susceptibility resulting in protein oxidation (McClements and Decker, 2000; Panpipat and Yongsawatdigul, 2008). Many studies have reported the incorporation of FO or PUFA in various foods, including bread, milk, meat, and ice cream (Muguerza et al., 2004; Valencia et al., 2006).

Oil-in-water $(\mathrm{O} / \mathrm{W})$ emulsions are efficient systems to 
disperse PUFA and thus help their incorporation into meat products. However, conventional $\mathrm{O} / \mathrm{W}$ emulsions show low resistance to oxidation stress due to a single layer and generate strong flavors in fish products, thus making it difficult to apply this system to the food industry. To prevent the drawback of oxidation, the FO emulsion system should be made multilayered, by including hydrocolloids such as chitosan and low methoxyl pectin in the preparation of the emulsion (Chun et al., 2013).

To mask the fishy flavor, it would be effective to add flavoring agents such as cinnamon oil. Based on previous research, trans-cinnamaldehyde (cinnamon oil; $\mathrm{CO}$ ) suppressed the fishy flavor of Thai fish sauce when added to the fish oil emulsion system (Choi et al., 2010). Alternate potential advantages of $\mathrm{CO}$ are its antimicrobial and antioxidative properties, which are applied to food packaging procedures (Becerril et al., 2007; Jo et al., 2015). It was hypothesized that $\mathrm{CO}$ would provide better storage stability in ground meat patties and reduce the fishy flavor caused by FO. Therefore, in this study, we aimed to evaluate the effect of multilayered FO emulsion systems containing cinnamon oil on the physicochemical properties and microbial stability of pork patties.

\section{Materials and Methods}

\section{Materials}

For multilayered FO preparation, FO and CO (98\%) were purchased from Sigma-Aldrich Chemical Company (USA). Polyoxyethylene sorbitan monolaurate (Tween
20), used as the emulsifier, was obtained from Amresco Inc. (USA). Chitosan from shrimp shells ( $>75 \%$ deacetylated according to the manufacturer's specification) and pectin from citrus peel (low methoxyl pectin) with galacturonic acid over $74 \%$ (dried basis) were chosen as biopolymers and were purchased from Sigma-Aldrich Chemical Company (USA). Fresh, lean pork meat and pork back fat were obtained from a local meat market to prepare the pork patties. All chemical products were of analytical grade.

\section{Preparation of multilayered FO}

Multilayered FO was formulated by the layer-by-layer (LbL) electrostatic deposition method described by Jo et al. (2012) and Chun et al. (2013), as depicted in Fig. 1. The composition of coating materials to prepare a multilayered FO $(1.0 \%, \mathrm{w} / \mathrm{w})$ without or with $\mathrm{CO}(0.05 \%, \mathrm{w} /$ w) included $1.25 \%(\mathrm{w} / \mathrm{w})$ Tween 20 (primary layer), $0.1 \%(\mathrm{w} / \mathrm{w})$ chitosan (secondary layer), and $0.2 \%(\mathrm{w} / \mathrm{w})$ low methoxyl pectin (tertiary layer).

\section{Pork patty preparation}

Fresh, lean pork meat and pork back fat were initially ground through an 8-mm plate. Seven batches were prepared differing in composition with respect to the type of multilayered emulsion and quantity added (Table 1). For each batch of patties, pork meat (75\%), pork back fat $(15 \%)$, cold water $(2 \%$ to $10 \%)$, multilayered emulsions ( $2 \%$ to $8 \%$ ), and sodium chloride $(\mathrm{NaCl}, 1.5 \%)$ were emulsified using a homomixer (5K5SS; USA) for $30 \mathrm{~min}$.

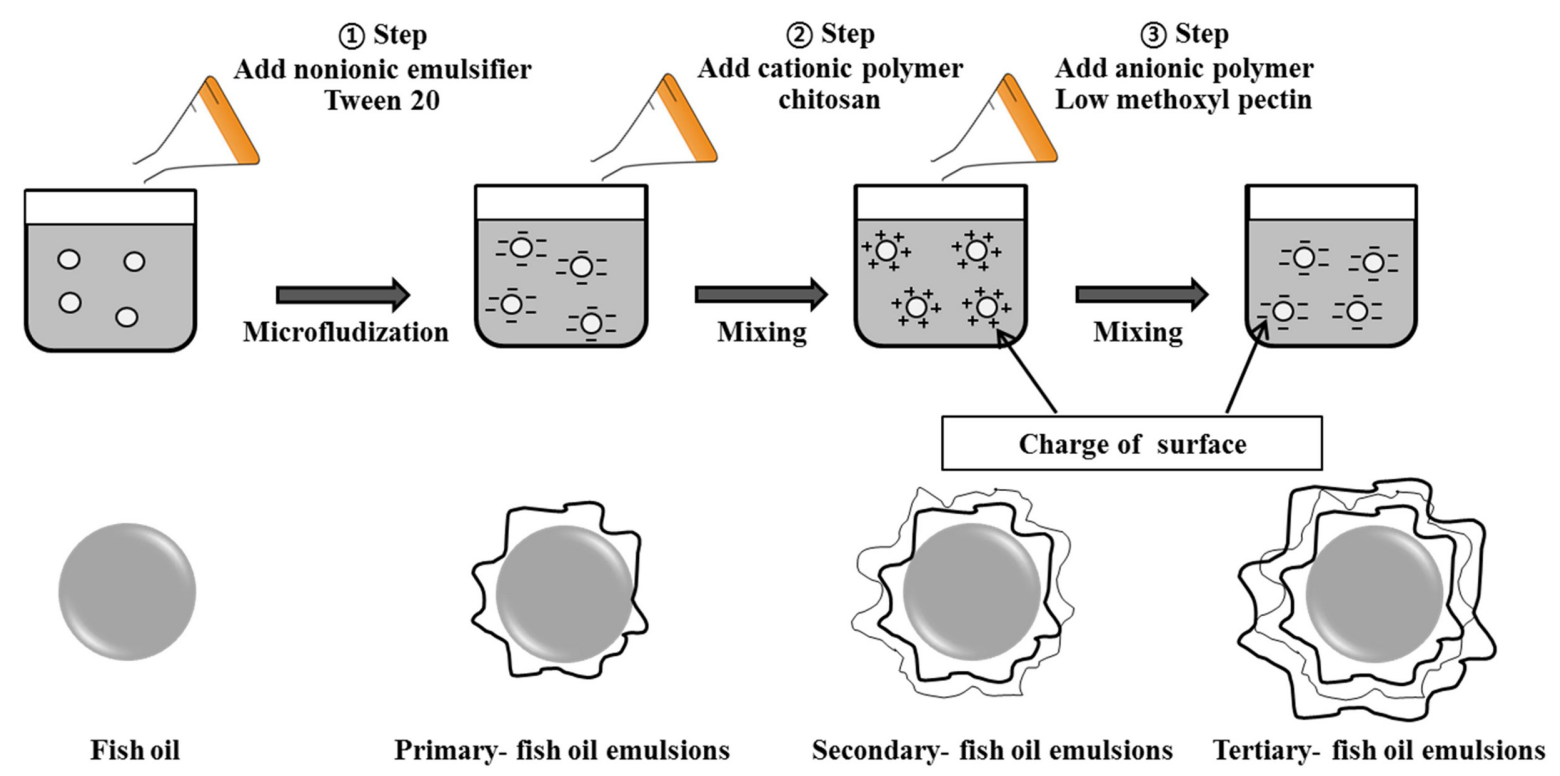

Fig. 1. Schematic of method of formulation of multilayered fish oil emulsions. 
Table 1. Formulation of pork patties with various levels of multilayered fish oil emulsions without or with cinnamon oil

\begin{tabular}{cccccccc}
\hline \hline \multirow{2}{*}{ Ingredients $(\%, w / w)$} & \multirow{2}{*}{ Control } & \multicolumn{6}{c}{ Treatments ${ }^{\text {I) }}$} \\
\cline { 3 - 7 } & & F1 & F2 & F3 & FC1 & FC2 & FC3 \\
\hline Pork meat & 75 & 75 & 75 & 75 & 75 & 75 & 75 \\
Pork fat & 15 & 15 & 15 & 15 & 15 & 15 & 15 \\
Cold water & 10 & 8 & 6 & 2 & 8 & 6 & 2 \\
Fish oil emulsions & 0 & 2 & 4 & 8 & 2 & 4 & 8 \\
Total & 100 & 100 & 100 & 100 & 100 & 100 & 100 \\
salt & 1.5 & 1.5 & 1.5 & 1.5 & 1.5 & 1.5 & 1.5 \\
\hline
\end{tabular}

${ }^{1)}$ Control, pork patties without fish oil emulsions; F1, pork patties with primary-fish oil (1\%) emulsion; F2, pork patties with secondaryfish oil $(0.5 \%)$ emulsion; F3, pork patties with tertiary-fish oil $(0.25 \%)$ emulsion; FC1, pork patties with primary-fish oil $(1 \%)$ emulsion added to cinnamon oil $(0.05 \%)$; FC2, pork patties with secondary-fish oil $(0.5 \%)$ emulsion added to cinnamon oil $(0.025 \%)$; FC3, pork patties with tertiary-fish oil $(0.25 \%)$ emulsion added to cinnamon oil $(0.0125 \%)$.

After emulsification, the samples weighed approximately $80 \mathrm{~g}$ and were placed in a Petri dish (100-mm-diameter, 15-mm-thick). The pork patties were packaged in thin polyethylene film with high oxygen permeability (equal to aerobic packaging) and then stored at $4^{\circ} \mathrm{C}$ until use. This procedure was performed in triplicate for each meat patty with multilayered FO. The quality and storage stability were determined after $0,5,10,15$, and $20 \mathrm{~d}$ at $5^{\circ} \mathrm{C}$.

\section{pH value}

A $5 \mathrm{~g}$ sample from each pork patty was taken, $50 \mathrm{~mL}$ distilled water was added and placed in a bag-filter (Interscience; France). The sample was blended using a stomacher (WS400, Shanghai Zhisun Equipment Co., Ltd.; China) for $3 \mathrm{~min}$. The $\mathrm{pH}$ of the filtrated solution was determined using a $\mathrm{pH}$ meter (Model S220, Mettler Toledo GmbH; Switzerland).

\section{Color evaluation}

The color of pork patties was determined using a colorimeter (Minolta Chroma meter CR-210; Japan) calibrated with a white standard (CIE L*=+97.83, CIE $a^{*=}$ -0.43 , CIE $\left.b^{*}=+1.96\right)$. The color measurement of pork patties was performed on the surface and measured 5 times in each pack at different locations. The color values of $L^{*}, a^{*}$, and $b^{*}$ were determined as indicators of lightness, redness, and yellowness, respectively. The total color difference $(\Delta \mathrm{E})$ between the control (at $0 \mathrm{~d}$ ) and pork patties containing multilayered FO was numerically calculated with the following equation:

$$
\Delta E=\sqrt{\Delta L^{*^{2}}+\Delta a^{*^{2}}+\Delta b^{*^{2}}}
$$

\section{Cooking loss}

A 5-cm-diameter and 1-cm-height sample (weight $20 \pm$ $0.5 \mathrm{~g}$ ) reconstructed from pork patties was placed into a polypropylene bag, cooked for $15 \mathrm{~min}$ at $75^{\circ} \mathrm{C}$ in a water bath, and cooled to room temperature. The cooking loss of cooked pork patties was determined by calculating the difference in weight before and after cooking as follows:

Cooking loss $(\%)=$ [weight of patty before cooking $(\mathrm{g})]$ - [weight of patty after cooking $(\mathrm{g}) /$ weight of patty before cooking $(\mathrm{g})] \times 100$

\section{Texture profile analysis (TPA)}

TPA was performed using a texture analyzer (CT3, Brookfield Engineering Labs Inc.; USA) equipped with a TA43 sphere 25.4-mm D (Brookfield Engineering Labs Inc.; USA). As mentioned above, pork patties were reconstructed to a $5-\mathrm{cm}$-diameter and 1-cm-height shape and cooked for $15 \mathrm{~min}$ at $75^{\circ} \mathrm{C}$ in a water bath. Before analysis, the cooked pork patties were kept to equilibrate to room temperature. The conditions of TPA were as follows: pre-test speed; $2.0 \mathrm{~mm} / \mathrm{s}$, post-test speed; $5.0 \mathrm{~mm} / \mathrm{s}$, test speed; $1.0 \mathrm{~mm} / \mathrm{s}$, maximum load; $1 \mathrm{~kg}$, distance; 8.0 $\mathrm{mm}$, force; $750 \mathrm{~g}$. Value for hardness $(\mathrm{kg})$, springiness (ratio), cohesiveness, gumminess $(\mathrm{kg})$, and chewiness $(\mathrm{kg})$ were determined.

\section{Thiobarbituric acid reactive substances (TBARS)}

The TBARS in pork patties were measured by the method of Hoyland and Taylor (1991). A $5 \mathrm{~g}$ sample was homogenized with $50 \mathrm{~mL}$ distilled water in a filter stomacher bag using a stomacher. A $0.75 \mathrm{~mL}$ aliquot of the filtrate was mixed with $2.5 \mathrm{~mL}$ benzene and $2.5 \mathrm{~mL} \mathrm{2-}$ thiobarbituric acid (TBA) reagent $(0.69 \%$ TBA solution mixed with equal amount of acetic acid) and centrifuged at $1500 \mathrm{~g}$ for $10 \mathrm{~min}$. After phase separation, the lower phase was filtered using a $0.45-\mu \mathrm{m}$ syringe filter (DISMIC, Advantec MFS, Inc.; Japan) and boiled for $30 \mathrm{~min}$ in a water bath at $95^{\circ} \mathrm{C}$. After cooling at ambient temper- 
ature, absorbance of the sample was measured at $530 \mathrm{~nm}$. The results were calculated as milligrams of malonaldehyde per kilogram of sample.

\section{Total viable count (TVC)}

For TVC analysis, a 5-g sample from each pork patty was aseptically homogenized in $45 \mathrm{~mL}$ sterile saline solution $(0.85 \% \mathrm{NaCl})$ in a filter stomacher bag using a stomacher for $3 \mathrm{~min}$. A $1-\mathrm{mL}$ aliquot of the filtrate was appropriately diluted using sterilized $0.85 \% \mathrm{NaCl}$ and plated on plate count agar (Sigma-Aldrich Co.; USA). After incubation at $37^{\circ} \mathrm{C}$ for $2 \mathrm{~d}$, plates with 30 to 300 visual colonies were selected and colonies were counted. Results are expressed as the logarithm of the number of colony-forming units ( $\log \mathrm{CFU} / \mathrm{g}$ ).

\section{Statistical analysis}

One-way analysis of variance (ANOVA) was performed using a SAS statistical program, ver. 9.2 (SAS Institute; USA); the means were separated by Duncan's multiple range test $(p<0.05)$, and correlations between independent variables and measured values were calculated using Pearson's correlation coefficients. All measurements were performed on at least 3 samples and are rep-

Table 2. Change in pH, cooking loss, water-holding capacity, and moisture content of pork patties with various levels of multilayered fish oil emulsions during refrigerated storage

\begin{tabular}{|c|c|c|c|c|c|}
\hline \multirow{2}{*}{ Treatments ${ }^{1)}$} & \multicolumn{5}{|c|}{ Storage periods $(\mathrm{d})$} \\
\hline & 0 & 5 & 10 & 15 & 20 \\
\hline \multicolumn{6}{|l|}{$\mathrm{pH}$} \\
\hline Control & $5.54 \pm 0.01^{\mathrm{Ba}^{*}}$ & $5.46 \pm 0.02^{\mathrm{Ab}}$ & $5.17 \pm 0.02^{\mathrm{Cc}}$ & $5.05 \pm 0.02^{\mathrm{Ce}}$ & $5.10 \pm 0.03^{\mathrm{Cd}}$ \\
\hline $\mathrm{F} 1$ & $5.53 \pm 0.04^{\mathrm{Ba}}$ & $5.45 \pm 0.02^{\mathrm{Ab}}$ & $5.14 \pm 0.01^{\mathrm{Cc}}$ & $5.05 \pm 0.02^{\mathrm{Cd}}$ & $5.14 \pm 0.02^{\mathrm{Dd}}$ \\
\hline $\mathrm{F} 2$ & $5.49 \pm 0.01^{\mathrm{Ca}}$ & $5.30 \pm 0.01^{\mathrm{Db}}$ & $5.16 \pm 0.01^{\mathrm{Cc}}$ & $5.08 \pm 0.03^{\mathrm{Cd}}$ & $5.18 \pm 0.06^{\mathrm{Dcd}}$ \\
\hline F3 & $5.58 \pm 0.01^{\mathrm{Aa}}$ & $5.40 \pm 0.01^{\mathrm{Bb}}$ & $5.25 \pm 0.01^{\mathrm{Ac}}$ & $5.15 \pm 0.03^{\mathrm{Bd}}$ & $5.19 \pm 0.03^{\mathrm{ABd}}$ \\
\hline $\mathrm{FC} 1$ & $5.60 \pm 1.09^{\mathrm{Aa}}$ & $5.40 \pm 0.01^{\mathrm{Bb}}$ & $5.21 \pm 0.00^{\mathrm{Bc}}$ & $5.15 \pm 0.02^{\mathrm{Bd}}$ & $5.23 \pm 0.08^{\mathrm{Bd}}$ \\
\hline $\mathrm{FC} 2$ & $5.46 \pm 0.01^{\mathrm{Da}}$ & $5.28 \pm 0.01^{\mathrm{Eb}}$ & $5.08 \pm 0.01^{\mathrm{Dc}}$ & $5.00 \pm 0.01^{\mathrm{Dd}}$ & $5.01 \pm 0.04^{\mathrm{Ed}}$ \\
\hline FC3 & $5.54 \pm 0.01^{\mathrm{Ba}}$ & $5.36 \pm 0.02^{\mathrm{Cb}}$ & $5.22 \pm 0.03^{\mathrm{ABc}}$ & $5.19 \pm 0.03^{\mathrm{Ac}}$ & $5.11 \pm 0.02^{\mathrm{Ac}}$ \\
\hline \multicolumn{6}{|c|}{ Cooking loss (\%) } \\
\hline Control & $15.88 \pm 1.93^{\mathrm{Bc}}$ & $19.48 \pm 1.36^{\mathrm{Cb}}$ & $17.95 \pm 0.29^{\mathrm{Cbc}}$ & $25.83 \pm 2.57^{\mathrm{Aa}}$ & $25.00 \pm 0.5^{\mathrm{ABa}}$ \\
\hline $\mathrm{F} 1$ & $17.22 \pm 0.83^{\mathrm{ABc}}$ & $18.71 \pm 0.17^{\mathrm{Cbc}}$ & $19.68 \pm 2.29^{\mathrm{ABb}}$ & $24.67 \pm 1.26^{\mathrm{Aa}}$ & $25.17 \pm 0.29^{\mathrm{ABa}}$ \\
\hline $\mathrm{F} 2$ & $19.19 \pm 1.01^{\mathrm{ACc}}$ & $22.06 \pm 0.68^{\mathrm{ABb}}$ & $23.28 \pm 1.48^{\mathrm{Aab}}$ & $25.17 \pm 1.04^{\mathrm{Aa}}$ & $25.17 \pm 1.04^{\mathrm{ABa}}$ \\
\hline F3 & $18.75 \pm 1.18^{\mathrm{Ad}}$ & $21.03 \pm 0.35^{\mathrm{ABc}}$ & $19.93 \pm 0.24^{\mathrm{BCDc}}$ & $26.17 \pm 0.76^{\mathrm{Aa}}$ & $23.17 \pm 1.04^{\mathrm{Bb}}$ \\
\hline $\mathrm{FC} 1$ & $18.16 \pm 1.46^{\mathrm{ACc}}$ & $19.53 \pm 1.08^{\mathrm{Cbc}}$ & $21.41 \pm 0.6^{\mathrm{ABbc}}$ & $25.17 \pm 1.26^{\mathrm{Aa}}$ & $24.50 \pm 0.5^{\mathrm{ABa}}$ \\
\hline $\mathrm{FC} 2$ & $18.93 \pm 0.61^{\mathrm{Ab}}$ & $20.93 \pm 0.69^{\mathrm{Bb}}$ & $19.67 \pm 0.89^{\mathrm{BCb}}$ & $26.33 \pm 3.4^{\mathrm{Aa}}$ & $25.67 \pm 1.04^{\mathrm{Aa}}$ \\
\hline FC3 & $18.47 \pm 0.92^{\mathrm{Ad}}$ & $22.40 \pm 0.25^{\mathrm{Ac}}$ & $21.99 \pm 0.21^{\mathrm{ABc}}$ & $25.83 \pm 1.26^{\mathrm{Aa}}$ & $24.33 \pm 0.29^{\mathrm{ABb}}$ \\
\hline \multicolumn{6}{|c|}{ Water holding capacity (\%) } \\
\hline Control & $79.79 \pm 3.01^{\mathrm{Aab}}$ & $83.48 \pm 5.6^{\mathrm{Aa}}$ & $81.48 \pm 0.63^{\mathrm{BCab}}$ & $76.04 \pm 5.33^{\mathrm{Ab}}$ & $74.91 \pm 0.83^{\mathrm{Db}}$ \\
\hline F1 & $80.57 \pm 2.97^{\mathrm{Abc}}$ & $86.10 \pm 3.63^{\mathrm{Aa}}$ & $82.93 \pm 2.47^{\mathrm{BCab}}$ & $76.23 \pm 0.83^{\text {Acd }}$ & $74.51 \pm 2.33^{\mathrm{Dd}}$ \\
\hline F2 & $81.90 \pm 3.51^{\text {Aab }}$ & $85.02 \pm 2.7^{\mathrm{Aa}}$ & $81.17 \pm 3.6^{\mathrm{BCab}}$ & $77.39 \pm 2.09^{\mathrm{Ab}}$ & $78.41 \pm 1.31^{\mathrm{BCb}}$ \\
\hline F3 & $78.82 \pm 5.67^{\mathrm{Ab}}$ & $88.57 \pm 2.48^{\mathrm{Aa}}$ & $87.00 \pm 1.06^{\mathrm{Aa}}$ & $85.12 \pm 4.28^{\mathrm{Aab}}$ & $84.98 \pm 0.52^{\text {Aab }}$ \\
\hline $\mathrm{FC} 1$ & $81.50 \pm 3.51^{\mathrm{Ab}}$ & $89.20 \pm 1.92^{\mathrm{Aa}}$ & $84.75 \pm 2.32^{\mathrm{ABab}}$ & $81.46 \pm 1.57^{\mathrm{Aab}}$ & $83.16 \pm 2.54^{\mathrm{Aab}}$ \\
\hline $\mathrm{FC} 2$ & $77.24 \pm 4.37^{\mathrm{Aa}}$ & $85.75 \pm 2.28^{\mathrm{Aa}}$ & $80.53 \pm 0.75^{\mathrm{Ca}}$ & $78.15 \pm 12.15^{\mathrm{Aa}}$ & $76.80 \pm 0.45^{\mathrm{CDa}}$ \\
\hline FC3 & $81.86 \pm 1.31^{\mathrm{Abc}}$ & $84.95 \pm 2.48^{\mathrm{Aa}}$ & $82.76 \pm 0.23^{\mathrm{BCab}}$ & $75.51 \pm 1.44^{\mathrm{Ac}}$ & $79.73 \pm 0.71^{\mathrm{Bc}}$ \\
\hline \multicolumn{6}{|c|}{ Moisture contents $(\%)$} \\
\hline Control & $64.58 \pm 2.87^{\mathrm{Aa}}$ & $67.40 \pm 0.35^{\text {Aa }}$ & $67.10 \pm 0.49^{\mathrm{Aa}}$ & $66.35 \pm 1.15^{\text {Aa }}$ & $64.21 \pm 2.47^{\mathrm{Aa}}$ \\
\hline F1 & $65.42 \pm 2.14^{\mathrm{Aab}}$ & $66.03 \pm 0.9 \mathrm{~A}^{\mathrm{Bab}}$ & $66.40 \pm 0.27^{\mathrm{ABa}}$ & $65.85 \pm 0.08^{\mathrm{Aab}}$ & $63.96 \pm 1.21^{\mathrm{Ab}}$ \\
\hline F2 & $65.23 \pm 1.22^{\mathrm{Ac}}$ & $67.45 \pm 0.11^{\mathrm{Aa}}$ & $67.08 \pm 0.49^{\mathrm{Aab}}$ & $65.73 \pm 0.26^{\mathrm{Abc}}$ & $65.20 \pm 1.11^{\mathrm{Ac}}$ \\
\hline F3 & $65.22 \pm 2.25^{\mathrm{Aa}}$ & $66.23 \pm 0.4 \mathrm{~A}^{\mathrm{Ba}}$ & $66.08 \pm 1.32^{\mathrm{ABCa}}$ & $66.19 \pm 0.72^{\mathrm{Aa}}$ & $64.62 \pm 2.88^{\mathrm{Aa}}$ \\
\hline $\mathrm{FC} 1$ & $65.47 \pm 1.31^{\mathrm{Aa}}$ & $64.90 \pm 1.01^{\mathrm{Ba}}$ & $65.53 \pm 0.74^{\mathrm{ABC}}$ & $65.74 \pm 0.41^{\mathrm{Aa}}$ & $65.29 \pm 0.97^{\mathrm{Aa}}$ \\
\hline $\mathrm{FC} 2$ & $64.34 \pm 1.72^{\mathrm{Aa}}$ & $65.72 \pm 1.58^{\mathrm{Ba}}$ & $64.50 \pm 1.68^{\mathrm{Ca}}$ & $65.74 \pm 1.59^{\mathrm{Aa}}$ & $66.03 \pm 0.69^{\mathrm{Aa}}$ \\
\hline FC3 & $64.50 \pm 1.48^{\mathrm{Aa}}$ & $65.33 \pm 0.83^{\mathrm{Ba}}$ & $65.24 \pm 0.71^{\mathrm{BCa}}$ & $64.89 \pm 0.53^{\mathrm{Aa}}$ & $65.62 \pm 0.59^{\mathrm{Aa}}$ \\
\hline
\end{tabular}

${ }^{1)}$ Control, pork patties without fish oil emulsions; F1, pork patties with primary-fish oil (1\%) emulsion; F2, pork patties with secondaryfish oil (0.5\%) emulsion; F3, pork patties with tertiary-fish oil $(0.25 \%)$ emulsion; FC1, pork patties with primary-fish oil ( $1 \%)$ emulsion added to cinnamon oil (0.05\%); FC2, pork patties with secondary-fish oil (0.5\%) emulsion added to cinnamon oil (0.025\%); FC3, pork patties with tertiary-fish oil $(0.25 \%)$ emulsion added to cinnamon oil $(0.0125 \%)$. * Mean \pm standard deviation of triplicate determinations $(n=3) .{ }^{A-G}$ Means with different superscripts within the same column are significantly different $(p<0.05)$. ${ }^{\mathrm{a}-\mathrm{e}}$ Means with different superscripts within the same row are significantly different $(p<0.05)$. 
orted as means with standard deviations (SD).

\section{Results and Discussion}

\section{Color}

Differences in values for lightness ( $\mathrm{L}^{*}$ value), redness ( $\mathrm{a}^{*}$ value), and yellowness ( $\mathrm{b}^{*}$ value) of pork patties containing multilayered FO were significantly affected by the type of emulsion added (Table 2). Lightness and yellowness in the control showed the lowest values. During storage, lightness values of all samples significantly increased, but yellowness values decreased $(p<0.05)$. However, the redness values of all samples were unchanged over the storage period $(p>0.05)$. Similar trends in redness values were previously shown by Serrano et al. (2006), where storage had no significant effect on the redness value of final meat products, nor were such values variable over the storage period. Discoloration is a major problem for final meat products due to the negative impact on consumer acceptance (Chen and Trout, 1991). The initial values for total color difference $(\Delta \mathrm{E})$ indices of all treated samples ranged from 5.34 to 6.67 , which showed no significant difference $(p>0.05)$ between treatments. However, $\Delta \mathrm{E}$ of all samples dynamically increased during refrigerated storage $(p<0.05)$; moreover, the $\Delta \mathrm{E}$ of samples containing multilayered FO were signifi-

Table 3. Change in CIE color of pork patties with various levels of multilayered fish oil emulsions during refrigerated storage

\begin{tabular}{|c|c|c|c|c|c|}
\hline \multirow{2}{*}{ Treatments ${ }^{1)}$} & \multicolumn{5}{|c|}{ Storage periods $(\mathrm{d})$} \\
\hline & 0 & 5 & 10 & 15 & 20 \\
\hline \multicolumn{6}{|l|}{ CIE L* } \\
\hline Control & $59.48 \pm 0.84^{\mathrm{Dd} *}$ & $59.61 \pm 0.754^{\mathrm{Dd}}$ & $63.17 \pm 0.792^{\mathrm{Dc}}$ & $64.59 \pm 0.854^{\mathrm{Db}}$ & $66.53 \pm 0.278^{\mathrm{Ba}}$ \\
\hline $\mathrm{F} 1$ & $65.87 \pm 1.149^{\mathrm{Ab}}$ & $64.06 \pm 0.565^{\mathrm{Cc}}$ & $67.15 \pm 1.013^{\mathrm{BCab}}$ & $67.90 \pm 0.726^{\mathrm{Bca}}$ & $68.30 \pm 0.465^{\mathrm{Aa}}$ \\
\hline $\mathrm{F} 2$ & $64.27 \pm 0.653^{\mathrm{BCd}}$ & $65.89 \pm 0.426^{\mathrm{Bc}}$ & $67.60 \pm 0.65^{\mathrm{BCb}}$ & $67.34 \pm 0.19^{\mathrm{Cb}}$ & $69.02 \pm 0.652^{\mathrm{Aa}}$ \\
\hline F3 & $65.65 \pm 0.656^{\mathrm{Abd}}$ & $66.69 \pm 0.431^{\mathrm{Abc}}$ & $69.21 \pm 0.639^{\mathrm{Ab}}$ & $69.26 \pm 0.442^{\mathrm{Ab}}$ & $68.52 \pm 0.399^{\mathrm{Aa}}$ \\
\hline $\mathrm{FC} 1$ & $64.58 \pm 0.295^{\mathrm{ABCc}}$ & $65.91 \pm 0.262^{\mathrm{Bb}}$ & $66.33 \pm 0.838^{\mathrm{Cb}}$ & $68.23 \pm 0.098^{\mathrm{ABCa}}$ & $68.08 \pm 0.715^{\mathrm{Aa}}$ \\
\hline $\mathrm{FC} 2$ & $65.39 \pm 0.623^{\mathrm{ABCc}}$ & $67.27 \pm 0.103^{\mathrm{Ab}}$ & $67.30 \pm 0.835^{\mathrm{BCb}}$ & $68.58 \pm 0.662^{\mathrm{ABa}}$ & $69.14 \pm 0.798^{\mathrm{Aa}}$ \\
\hline FC3 & $64.22 \pm 0.641^{\mathrm{Cb}}$ & $66.36 \pm 0.44^{\mathrm{Bc}}$ & $68.59 \pm 0.711^{\mathrm{ABa}}$ & $68.40 \pm 0.407^{\mathrm{ABa}}$ & $68.50 \pm 1.055^{\mathrm{Aa}}$ \\
\hline \multicolumn{6}{|l|}{ CIE a* } \\
\hline Control & $7.44 \pm 0.298^{\mathrm{Bb}}$ & $8.60 \pm 0.198^{\mathrm{Aa}}$ & $7.11 \pm 0.395^{\mathrm{Cb}}$ & $7.83 \pm 0.913^{\mathrm{ABab}}$ & $7.44 \pm 0.298^{\mathrm{Abb}}$ \\
\hline $\mathrm{F} 1$ & $5.69 \pm 0.603^{\mathrm{Db}}$ & $7.73 \pm 0.591^{\mathrm{Bca}}$ & $7.67 \pm 0.213^{\mathrm{Aba}}$ & $7.85 \pm 0.148^{\mathrm{Aa}}$ & $7.49 \pm 0.322^{\mathrm{Aa}}$ \\
\hline $\mathrm{F} 2$ & $6.55 \pm 0.23^{\mathrm{Cc}}$ & $7.36 \pm 0.099^{\mathrm{Cb}}$ & $7.97 \pm 0.216^{\mathrm{Aa}}$ & $7.74 \pm 0.088^{\mathrm{ABab}}$ & $6.70 \pm 0.347^{\mathrm{Bc}}$ \\
\hline F3 & $7.50 \pm 0.038^{\mathrm{Ba}}$ & $7.39 \pm 0.384^{\mathrm{Cab}}$ & $7.36 \pm 0.115^{\mathrm{BCab}}$ & $6.82 \pm 0.372^{\mathrm{Cc}}$ & $6.98 \pm 0.086^{\mathrm{ABbc}}$ \\
\hline $\mathrm{FC} 1$ & $6.40 \pm 0.07^{\mathrm{Cc}}$ & $7.33 \pm 0.195^{\mathrm{Cab}}$ & $7.54 \pm 0.22^{\mathrm{ABCa}}$ & $7.08 \pm 0.437^{\mathrm{ABCab}}$ & $6.95 \pm 0.352^{\mathrm{ABb}}$ \\
\hline $\mathrm{FC} 2$ & $6.96 \pm 0.173^{\mathrm{BCb}}$ & $8.01 \pm 0.115^{\mathrm{Ba}}$ & $7.99 \pm 0.367^{\mathrm{Aa}}$ & $7.61 \pm 0.516^{\mathrm{ABCa}}$ & $6.68 \pm 0.268^{\mathrm{Bb}}$ \\
\hline FC3 & $8.32 \pm 0.591^{\mathrm{Aa}}$ & $6.40 \pm 0.204^{\mathrm{Dc}}$ & $7.24 \pm 0.127^{\mathrm{BCb}}$ & $6.94 \pm 0.255^{\mathrm{BCbc}}$ & $7.00 \pm 0.484^{\mathrm{Abbc}}$ \\
\hline \multicolumn{6}{|l|}{$\mathrm{CIE} \mathrm{b}^{*}$} \\
\hline Control & $8.14 \pm 0.038^{\mathrm{Ba}}$ & $8.18 \pm 0.251^{\mathrm{Ca}}$ & $5.95 \pm 0.229^{\mathrm{Cc}}$ & $7.10 \pm 0.163^{\mathrm{Cb}}$ & $6.13 \pm 0.378^{\mathrm{Cc}}$ \\
\hline $\mathrm{F} 1$ & $8.76 \pm 0.455^{\mathrm{Ba}}$ & $8.64 \pm 0.66 \mathrm{~B}^{\mathrm{Ca}}$ & $7.94 \pm 0.601^{\mathrm{Ba}}$ & $8.66 \pm 0.527^{\mathrm{Aa}}$ & $8.43 \pm 0.362^{\mathrm{Aa}}$ \\
\hline $\mathrm{F} 2$ & $10.28 \pm 0.128^{\mathrm{Aa}}$ & $9.46 \pm 0.411^{\mathrm{Abb}}$ & $8.14 \pm 0.605^{\mathrm{Bc}}$ & $8.29 \pm 0.132^{\mathrm{Abc}}$ & $7.90 \pm 0.074^{\mathrm{Abc}}$ \\
\hline F3 & $9.93 \pm 0.416^{\mathrm{Aa}}$ & $8.37 \pm 0.297^{\mathrm{Cb}}$ & $8.18 \pm 0.386^{\mathrm{Bbc}}$ & $7.72 \pm 0.267^{\mathrm{BCc}}$ & $7.60 \pm 0.146^{\mathrm{Bbc}}$ \\
\hline FC1 & $9.86 \pm 0.198^{\mathrm{Aa}}$ & $8.29 \pm 0.238^{\mathrm{Cab}}$ & $8.51 \pm 0.151^{\mathrm{Abb}}$ & $7.56 \pm 0.279^{\mathrm{Cac}}$ & $7.28 \pm 0.19^{\mathrm{Bc}}$ \\
\hline $\mathrm{FC} 2$ & $10.30 \pm 0.427^{\mathrm{Aa}}$ & $9.26 \pm 0.168^{\mathrm{ABb}}$ & $9.02 \pm 0.464^{\mathrm{Ab}}$ & $8.87 \pm 0.557^{\mathrm{Ab}}$ & $7.74 \pm 0.426^{\mathrm{Bc}}$ \\
\hline FC3 & $10.21 \pm 0.821^{\mathrm{Aa}}$ & $9.76 \pm 0.811^{\mathrm{Aa}}$ & $8.20 \pm 0.076^{\mathrm{Bbc}}$ & $8.65 \pm 0.332^{\mathrm{Ab}}$ & $7.25 \pm 0.596^{\mathrm{Bc}}$ \\
\hline \multicolumn{6}{|c|}{ Total color difference $(\Delta \mathrm{E})$} \\
\hline Control & & $1.34 \pm 0.05^{\mathrm{Fc}}$ & $4.34 \pm 0.71^{\mathrm{Db}}$ & $5.30 \pm 0.73^{\mathrm{Db}}$ & $7.35 \pm 0.36^{\mathrm{Ba}}$ \\
\hline $\mathrm{F} 1$ & $6.67 \pm 1.27^{\mathrm{Ab}}$ & $4.68 \pm 0.46^{\mathrm{Ec}}$ & $7.70 \pm 0.99^{\mathrm{BCab}}$ & $8.46 \pm 0.75^{\mathrm{BCa}}$ & $8.83 \pm 0.45^{\mathrm{Aa}}$ \\
\hline $\mathrm{F} 2$ & $5.34 \pm 0.59^{\mathrm{Ad}}$ & $6.55 \pm 0.49^{\mathrm{CDc}}$ & $8.16 \pm 0.64^{\mathrm{BCb}}$ & $7.88 \pm 0.19^{\mathrm{Cb}}$ & $9.58 \pm 0.68^{\mathrm{Aa}}$ \\
\hline F3 & $6.44 \pm 0.71^{\mathrm{Ab}}$ & $7.23 \pm 0.42^{\mathrm{Bb}}$ & $9.74 \pm 0.64^{\mathrm{Aa}}$ & $9.82 \pm 0.45^{\mathrm{Aa}}$ & $9.08 \pm 0.40^{\mathrm{Aa}}$ \\
\hline $\mathrm{FC} 1$ & $5.49 \pm 0.29^{\mathrm{Ac}}$ & $6.45 \pm 0.26^{\mathrm{Db}}$ & $6.87 \pm 0.83^{\mathrm{Cb}}$ & $8.79 \pm 0.10^{\mathrm{BCa}}$ & $8.67 \pm 0.68^{\mathrm{Aa}}$ \\
\hline $\mathrm{FC} 2$ & $6.34 \pm 0.42^{\mathrm{Ac}}$ & $7.90 \pm 0.09^{\mathrm{Ab}}$ & $7.91 \pm 0.82^{\mathrm{BCb}}$ & $9.15 \pm 0.64^{\mathrm{ABa}}$ & $9.71 \pm 0.82^{\mathrm{Aa}}$ \\
\hline $\mathrm{FC} 3$ & $5.29 \pm 0.87^{\mathrm{Ac}}$ & $7.18 \pm 0.48^{\mathrm{BCb}}$ & $9.12 \pm 0.71^{\mathrm{ABa}}$ & $8.96 \pm 0.38^{\mathrm{ABa}}$ & $9.10 \pm 1.03^{\mathrm{Aa}}$ \\
\hline
\end{tabular}

${ }^{1)}$ Control, pork patties without fish oil emulsions; F1, pork patties with primary-fish oil (1\%) emulsion; F2, pork patties with secondaryfish oil (0.5\%) emulsion; F3, pork patties with tertiary-fish oil $(0.25 \%)$ emulsion; FC1, pork patties with primary-fish oil $(1 \%)$ emulsion added to cinnamon oil (0.05\%); FC2, pork patties with secondary-fish oil $(0.5 \%)$ emulsion added to cinnamon oil $(0.025 \%)$; FC3, pork patties with tertiary-fish oil $(0.25 \%)$ emulsion added to cinnamon oil $(0.0125 \%)$. * Mean \pm standard deviation of triplicate determinations $(\mathrm{n}=3) .{ }^{\mathrm{A}-\mathrm{G}}$ Means with different superscripts within the same column are significantly different $(p<0.05) .{ }^{\mathrm{a}-\mathrm{e}}$ Means with different superscripts within the same row are significantly different $(p<0.05)$. 
cantly higher than that of control samples during storage. Therefore, we concluded that the color of samples showed no significant difference with layered FO, whereas the color changed during storage.

\section{pH, cooking loss, water-holding capacity, moisture content}

Table 3 shows the results for $\mathrm{pH}$ values of pork patties containing multilayered FO during storage. The $\mathrm{pH}$ values of all samples significantly decreased from 5.60 to 5.00 during storage. $\mathrm{F} 2$ and $\mathrm{FC} 2$ had lower $\mathrm{pH}$ values than the control, F1, F3, FC1, and FC3. During storage, the $\mathrm{pH}$ consistently decreased during storage by $20 \mathrm{~d}$, similar to $\mathrm{pH}$ trends previously observed by Choi et al. (2011) when different amounts of lotus leaf powder were added to chicken patties during refrigerated storage over $28 \mathrm{~d}$. According to Kim et al. (2007), the $\mathrm{pH}$ value of ground pork meat containing paprika significantly decreased.

Cooking loss in pork patties containing multilayered FO during storage is presented in Table 3 . The fresh pork patties had the lowest cooking loss owing to low water and fat content. During storage, cooking loss consistently increased for all samples $(p<0.05)$. On the other hand, water-holding capacity and moisture content were unchanged. Razminowicz et al. (2006) reported that cooking loss of meat was related to water-holding capacity, defined as the ability of meat to retain water during exposure to external forces such as cutting, heating, grinding, and pressing, and is an important factor to determining the juiciness of meat (Zhang et al., 2005). Furthermore, Hutchings (1999) reported that moisture content may be relevant to the lightness value of color in food. However, in our study, moisture content was not affected although lightness of color in the pork patties increased during storage. Therefore, we suggest that multilayered FO can be applied to pork patties without loss of meat quality.

\section{TPA}

Texture properties of the pork patty samples containing multilayered FO are presented in Table 4. No significant

Table 4. Change in hardness, cohesiveness, and springiness of pork patties with various levels of multilayered fish oil emulsions during refrigerated storage

\begin{tabular}{|c|c|c|c|c|c|}
\hline \multirow{2}{*}{ Treatments $^{1)}$} & \multicolumn{5}{|c|}{ Storage periods (d) } \\
\hline & 0 & 5 & 10 & 15 & 20 \\
\hline \multicolumn{6}{|c|}{ Hardness (g) } \\
\hline Control & $1532 \pm 29^{b^{*}}$ & $1747 \pm 117^{\mathrm{a}}$ & $1466 \pm 202^{\mathrm{ab}}$ & $1643 \pm 135^{\mathrm{ab}}$ & $2081 \pm 197^{\mathrm{ab}}$ \\
\hline $\mathrm{F} 1$ & $1467 \pm 134^{\mathrm{b}}$ & $1753 \pm 100^{\mathrm{ab}}$ & $1611 \pm 139^{\mathrm{ab}}$ & $1555 \pm 91^{\mathrm{ab}}$ & $1686 \pm 110^{\mathrm{a}}$ \\
\hline $\mathrm{F} 2$ & $1334 \pm 89^{b}$ & $1540 \pm 121^{\mathrm{ab}}$ & $1581 \pm 213^{b}$ & $1625 \pm 121^{\mathrm{ab}}$ & $1828 \pm 238^{a b}$ \\
\hline F3 & $1418 \pm 180^{\mathrm{a}}$ & $1669 \pm 50^{\mathrm{a}}$ & $1467 \pm 190^{\mathrm{a}}$ & $1638 \pm 116^{\mathrm{a}}$ & $1894 \pm 40^{\mathrm{a}}$ \\
\hline $\mathrm{FC} 1$ & $1579 \pm 245^{\mathrm{a}}$ & $1621 \pm 152^{\mathrm{a}}$ & $1550 \pm 82^{\mathrm{a}}$ & $1770 \pm 77^{\mathrm{a}}$ & $1831 \pm 69^{a}$ \\
\hline $\mathrm{FC} 2$ & $1290 \pm 166^{b}$ & $1699 \pm 165^{\mathrm{a}}$ & $1377 \pm 149^{b}$ & $1751 \pm 355^{\mathrm{a}}$ & $1722 \pm 348^{a}$ \\
\hline $\mathrm{FC} 3$ & $1445 \pm 159^{\mathrm{a}}$ & $1869 \pm 79^{a}$ & $1483 \pm 166^{\mathrm{a}}$ & $1828 \pm 79^{a}$ & $1988 \pm 260^{\mathrm{a}}$ \\
\hline \multicolumn{6}{|c|}{ Cohesiveness } \\
\hline Control & $0.60 \pm 0.012^{\mathrm{a}}$ & $0.59 \pm 0.015^{\mathrm{a}}$ & $0.54 \pm 0.038^{\mathrm{b}}$ & $0.55 \pm 0.038^{\mathrm{b}}$ & $0.56 \pm 0.023^{b}$ \\
\hline $\mathrm{F} 1$ & $0.58 \pm 0.017^{\mathrm{a}}$ & $0.58 \pm 0^{\mathrm{a}}$ & $0.53 \pm 0.017^{\mathrm{a}}$ & $0.51 \pm 0.046^{\mathrm{a}}$ & $0.50 \pm 0.02^{\mathrm{a}}$ \\
\hline $\mathrm{F} 2$ & $0.55 \pm 0.053^{\mathrm{a}}$ & $0.59 \pm 0.089^{\mathrm{ab}}$ & $0.52 \pm 0.015^{\mathrm{b}}$ & $0.49 \pm 0.049^{\mathrm{ab}}$ & $0.53 \pm 0.055^{\mathrm{ab}}$ \\
\hline F3 & $0.58 \pm 0.025^{\mathrm{a}}$ & $0.55 \pm 0.049^{\mathrm{a}}$ & $0.50 \pm 0.075^{\mathrm{b}}$ & $0.57 \pm 0.006^{\mathrm{b}}$ & $0.56 \pm 0.026^{\mathrm{ab}}$ \\
\hline $\mathrm{FC} 1$ & $0.58 \pm 0.012^{\mathrm{a}}$ & $0.61 \pm 0.025^{\mathrm{a}}$ & $0.51 \pm 0.061^{\mathrm{a}}$ & $0.51 \pm 0.025^{\mathrm{a}}$ & $0.56 \pm 0.026^{\mathrm{a}}$ \\
\hline $\mathrm{FC} 2$ & $0.55 \pm 0.026^{\mathrm{a}}$ & $0.59 \pm 0.015^{\mathrm{ab}}$ & $0.35 \pm 0.309^{b c}$ & $0.53 \pm 0.053^{b c}$ & $0.51 \pm 0.055^{\mathrm{c}}$ \\
\hline FC3 & $0.60 \pm 0.006^{\mathrm{a}}$ & $0.58 \pm 0.015^{\mathrm{a}}$ & $0.53 \pm 0.035^{\mathrm{b}}$ & $0.52 \pm 0.021^{\mathrm{b}}$ & $0.51 \pm 0.064^{\mathrm{b}}$ \\
\hline \multicolumn{6}{|c|}{ Springiness (mm) } \\
\hline Control & $3.45 \pm 0.112^{\mathrm{ab}}$ & $3.74 \pm 0.047^{\mathrm{ab}}$ & $3.32 \pm 0.295^{\mathrm{b}}$ & $3.67 \pm 0.091^{\mathrm{ab}}$ & $3.66 \pm 0.101^{\mathrm{ab}}$ \\
\hline F1 & $3.33 \pm 0.143^{\mathrm{b}}$ & $3.62 \pm 0.029^{\mathrm{a}}$ & $3.47 \pm 0.074^{\mathrm{ab}}$ & $3.49 \pm 0.068^{\mathrm{ab}}$ & $3.50 \pm 0.139^{\mathrm{ab}}$ \\
\hline $\mathrm{F} 2$ & $3.46 \pm 0.268^{\mathrm{a}}$ & $3.61 \pm 0.186^{\mathrm{a}}$ & $3.48 \pm 0.092^{\mathrm{a}}$ & $3.54 \pm 0.081^{\mathrm{a}}$ & $3.53 \pm 0.105^{\mathrm{a}}$ \\
\hline F3 & $3.52 \pm 0.121^{\mathrm{a}}$ & $3.55 \pm 0.084^{\mathrm{a}}$ & $3.50 \pm 0.24^{\mathrm{a}}$ & $3.62 \pm 0.2^{\mathrm{a}}$ & $3.67 \pm 0.096^{\mathrm{a}}$ \\
\hline $\mathrm{FC} 1$ & $3.55 \pm 0.121^{\mathrm{bc}}$ & $3.74 \pm 0.1^{\mathrm{a}}$ & $3.55 \pm 0.071^{\mathrm{bc}}$ & $3.49 \pm 0.056^{\mathrm{c}}$ & $3.69 \pm 0.056^{\mathrm{ab}}$ \\
\hline $\mathrm{FC} 2$ & $3.44 \pm 0.118^{b}$ & $3.75 \pm 0.045^{\mathrm{a}}$ & $3.39 \pm 0.136^{\mathrm{b}}$ & $3.57 \pm 0.185^{\mathrm{ab}}$ & $3.55 \pm 0.159^{\mathrm{ab}}$ \\
\hline FC3 & $3.43 \pm 0.127 \mathrm{ab}$ & $3.64 \pm 0.055 \mathrm{a}$ & $3.36 \pm 0.127 b$ & $3.69 \pm 0.139 \mathrm{a}$ & $3.50 \pm 0.2 \mathrm{ab}$ \\
\hline
\end{tabular}

${ }^{1)}$ Control, pork patties without fish oil emulsions; F1, pork patties with primary-fish oil (1\%) emulsion; F2, pork patties with secondaryfish oil $(0.5 \%)$ emulsion; F3, pork patties with tertiary-fish oil $(0.25 \%)$ emulsion; FC1, pork patties with primary-fish oil $(1 \%)$ emulsion added to cinnamon oil (0.05\%); FC2, pork patties with secondary-fish oil (0.5\%) emulsion added to cinnamon oil (0.025\%); FC3, pork patties with tertiary-fish oil $(0.25 \%)$ emulsion added to cinnamon oil $(0.0125 \%)$. *Mean \pm standard deviation of triplicate determinations $(n=3) .{ }^{A-G}$ Means with different superscripts within the same column are significantly different $(p<0.05){ }^{{ }^{a-e}}{ }^{\text {Means }}$ with different superscripts within the same row are significantly different $(p<0.05)$. 
change in hardness, cohesiveness, and springiness were observed between the control and pork patties containing multilayered FO during refrigerated storage. Ziegler et al. (1987) reported that texture characteristics of sausages were related to moisture level; for example, increase in moisture content causes decrease in hardness and increase in springiness of sausages. Moreover, they suggested that the moisture content had no effect on cohesiveness. The results of this study suggest that multilayered FO did not significantly affect the texture characteristics of the pork patties.

\section{Lipid oxidation}

Fig. 2 shows the TBARS values of pork patties containing multilayered FO during storage. The TBARS values of pork patties containing multilayered FO were significantly higher than those of the control $(p<0.05)$, while the TBARS values of all samples generally increa- sed during refrigerated storage. At $20 \mathrm{~d}$ of storage, TBARS values of the control, F1, F2, F3, FC1, FC2, and FC3 were 2.14, $6.53,5.80,4.54,5.08,4.85$, and $4.44 \mathrm{mg}$ malonaldehyde/ $\mathrm{kg}$, respectively. In our study, the TBARS value was remarkably decreased as the coating layer number increased $(p<0.05)$. It was thought that with fewer coating layers, free oil (FO, CO) would be released more quickly in emulsions. In addition, little antioxidant activity was observed in pork patties containing multilayered $\mathrm{FO}$ with CO (Fig. 2B). Namely, F1, F2, and F3 showed higher oxidation than $\mathrm{FC} 1, \mathrm{FC} 2$, and $\mathrm{FC} 3$ owing to addition of CO. According to López-López et al. (2010), lower antioxidant activity was required from seaweed due to the low level of lipid oxidation in the product. Other researchers reported that when olive oil was partially replaced by pork back fat no oxidation problems were encountered in fermented pork sausage.

\section{TVC}

Results of the TVC analysis of the pork patties containing multilayered FO during refrigerated storage are presented in Fig. 3. TVC of fresh patties significantly increased from 5.00 Log CFU/g to $6.46 \mathrm{Log} \mathrm{CFU} / \mathrm{g}$ during storage, and pork patties containing multilayered $\mathrm{FO}$ were also constantly increased. At $20 \mathrm{~d}$ of storage, the TVC of control samples was 6.46 Log CFU/g and that of treated samples F1, F2, F3, FC1, FC2, and FC3 was 6.43, 6.42, $5.88,6.18,6.48$, and $5.96 \mathrm{Log} \mathrm{CFU} / \mathrm{g}$, respectively. In general, when the total aerobic bacteria counts in meat reached 7.0 Log CFU/g, corruption and off-odor occurred (Egan et al., 1980). Analyzing the stored pork patties after
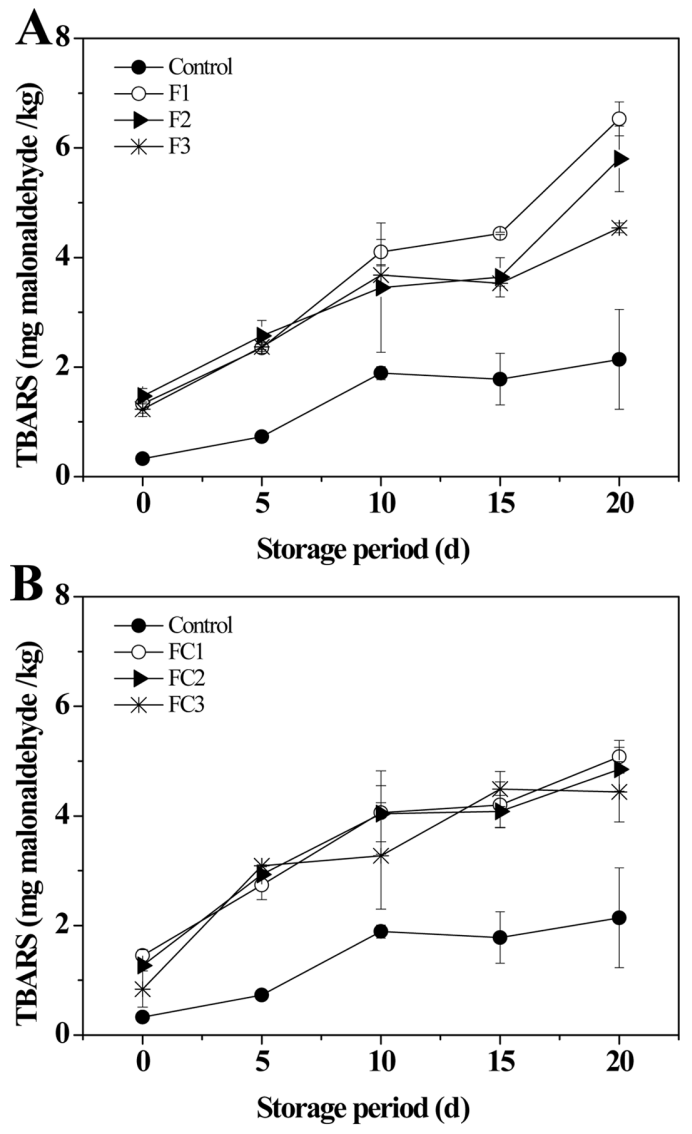

Fig. 2. Change in TBARS of pork patties with various levels of multilayered fish oil emulsions during refrigerated storage. Control, pork patties without fish oil emulsions; F1, pork patties with primary fish oil (1\%) emulsion; F2, pork patties with secondary fish oil $(0.5 \%)$ emulsion; $\mathrm{F} 3$, pork patties with tertiary fish oil $(0.25 \%)$ emulsion; FC1, pork patties with primary fish oil $(1 \%)$ emulsion added to cinnamon oil $(0.05 \%)$; FC2, pork patties with secondary fish oil $(0.5 \%)$ emulsion added to cinnamon oil $(0.025 \%)$; FC3, pork patties with tertiary fish oil $(0.25 \%)$ emulsion added to cinnamon oil $(0.0125 \%)$.

$20 \mathrm{~d}$, the TVC of F3 (5.88 Log CFU/g) and FC3 (5.96 Log CFU/g) was significantly lower than those of F1, FC1, F2 and FC2. Namely, deterioration of F3 and FC3 was occurred less than that of other samples. Because the F3 and FC3 showed inhibition of microbial proliferation, these were safe from bacteria during storage. In addition, these did not remarkable change with addition of $\mathrm{CO}$, probably since only a small amount of $\mathrm{CO}$ was used in this study. López-López et al. (2010) studied the characteristics of beef patties with low fat by adding olive oil to water emulsions. They reported that microbial populations generally increased with addition of the seaweed Wakame, and decreased with addition of olive oil. Therefore, the multilayered FO could be extended the storage 

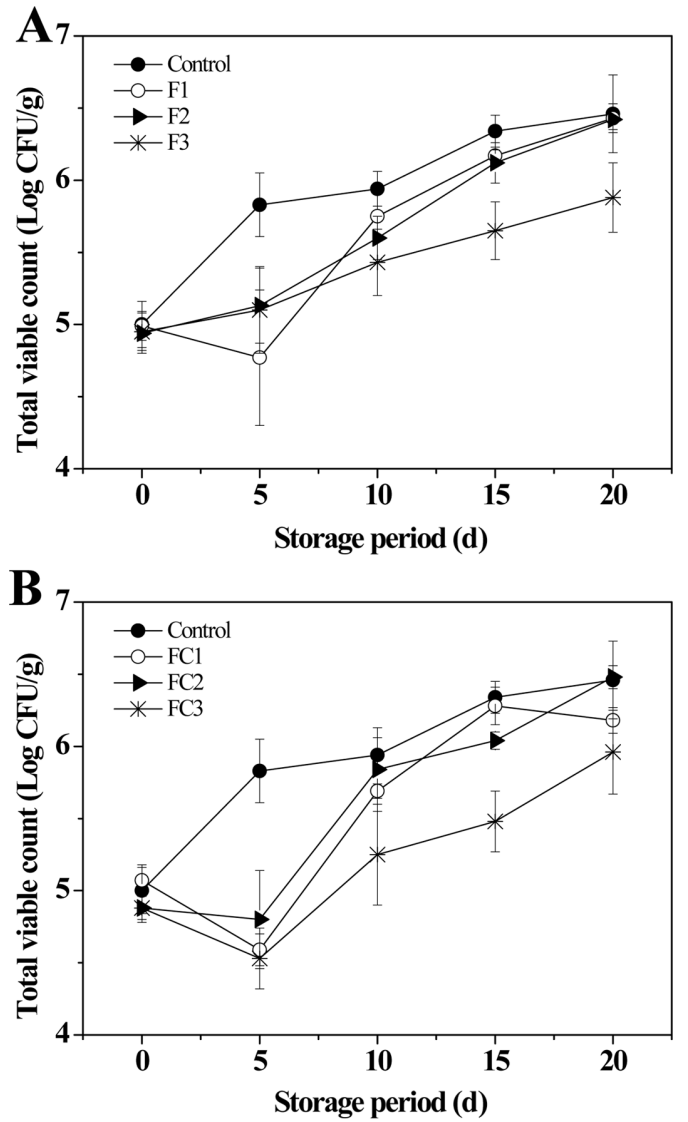

Fig. 3. Change in total viable count of pork patties with various levels of multilayered fish oil emulsions during refrigerated storage. Control, pork patties without fish oil emulsions; F1, pork patties with primary fish oil (1\%) emulsion; F2, pork patties with secondary fish oil $(0.5 \%)$ emulsion; F3, pork patties with tertiary fish oil $(0.25 \%)$ emulsion; FC1, pork patties with primary fish oil $(1 \%)$ emulsion added to cinnamon oil $(0.05 \%)$; FC2, pork patties with secondary fish oil $(0.5 \%)$ emulsion added to cinnamon oil $(0.025 \%)$; FC3, pork patties with tertiary fish oil $(0.25 \%)$ emulsion added to cinnamon oil $(0.0125 \%)$.

periods by inhibiting deterioration of meat products by microorganisms.

\section{Conclusion}

In this study, the addition of multilayered FO to pork patties was shown to provide high-quality fat and at the same time did not influence physical and textural changes during storage. However, the pork patties made with addition of multilayered FO had significantly higher TBARS values as compared to the control. Moreover, the effects of $\mathrm{CO}$ had no remarkable difference in pork patties. Thus, in order to improve the shelf-life and commercial application in the meat industry, further study is required to re- duce oxidation of fats and oils in meat and meat products. To maintain the organoleptic properties of meat products, a sensory test is also required.

\section{Acknowledgements}

This study was supported by a grant from the Korea Institute of Planning and Evaluation for Technology in Food, Agriculture, Forestry, and Fisheries (iPET Project No. 112022-3).

\section{References}

1. Becerril, R., Gómez-Lus, R., Goñi, P., López, P., and Nerín, C. (2007) Combination of analytical and microbiological techniques to study the antimicrobial activity of a new active food packaging containing cinnamon or oregano against $E$. coli and S. aureus. Anal. Bioanal. Chem. 388, 1003-1011.

2. Chen, C. M. and Trout, G. R. (1991) Sensory, instrumental texture profile and cooking properties of restructured beef steaks made with various binders. J. Food Sci. 56, 14571460 .

3. Choi, M. J., Ruktanonchai, U., Min, S. G., Chun, J. Y., and Soottitantawat, A. (2010) Physical characteristics of fish oil encapsulated by $\beta$-cyclodextrin using an aggregation method or polycaprolactone using an emulsion-diffusion method. Food Chem. 119, 1694-1703.

4. Choi, Y. S., Choi, J. H., Kim, H. Y., Kim, H. W., Lee, M. A., Chung, H. J., Lee, S. K., and Kim, C. J. (2011) Effect of lotus (Nelumbo nucifera) leaf powder on the quality characteristics of chicken patties in refrigerated storage. Korean J. Food Sci. An. 31, 9-18.

5. Chun, J. Y., Choi, M. J., Min, S. G., and Weiss, J. (2013) Formation and stability of multiple-layered liposomes by layerby-layer electrostatic deposition of biopolymers. Food Hydrocoll. 30, 249-257.

6. Colmenero, F. J. (1996) Technologies for developing low-fat meat products. Trends Food Sci. Tech. 7, 41-48.

7. Egan, A. F., Ford, A. L., and Shay, B. J. (1980) A comparison of Brochothrix thermosphacta and lactobacilli as a spoilage organism of vacuum packaged sliced luncheon meats. $J$. Food Sci. 45, 1745-1748.

8. Hoyland, D. V. and Taylor, A. J. (1991) A review of the methodology of the 2-thiobarbituric acid test. Food Chem. 40, 271-291.

9. Hutchings, J. B. (1999) Food color and appearance. Aspen Publishers Inc., Maryland, pp. 453-541.

10. Jiménez-Colmenero, F., Carballo, J., and Cofrades, S. (2001) Healthier meat and meat products: their role as functional foods. Meat Sci. 59, 5-13.

11. Jo, Y. J., Chun, J. Y., Kwon, Y. J., Min, S. G., Hong, G. P., and Choi, M. J. (2015) Physical and antimicrobial properties of trans-cinnamaldehyde nanoemulsions in water melon juice. LWT - Food Sci. Tech. 60, 444-451. 
12. Jo, Y. J., Lee, S. B., Kwon, Y. J., and Choi, M. J. (2012) Multi-layered encapsulation of fish oil through layer-bylayer electrostatic interaction. Abstracts, The 79th annual meeting of Korean Society of Food Sci. Technol. Dajoen, Korea.

13. Josquin, N. M., Linssen, J. P. H., and Houben, J. H. (2012) Quality characteristics of Dutch-style fermented sausages manufactured with partial replacement of pork back-fat with pure, pre-emulsified or encapsulated fish oil. Meat Sci. 90, 81-86.

14. Kim, S. J., Choi, W. S., You, S. G., and Min, Y. S. (2007) Effect of glucomannan on quality and shelf-life of low-fat chicken patty. Korean J. Food Sci. Technol. 39, 55-60.

15. López-López, I., Cofrades, S., Yakan, A., Solas, M. T., and Jiménez-Colmenero, F. (2010) Frozen storage characteristics of low-salt and low-fat beef patties as affected by Wakame addition and replacing pork backfat with olive oil-in-water emulsion. Food Res. Int. 43, 1244-1254.

16. Lurueña-Martýìnez, M. A., Vivar-Quintana, A. M., and Revilla, I. (2004) Effect of locust bean/xanthan gum addition and replacement of pork fat with olive oil on the quality characteristics of low-fat frankfurters. Meat Sci. 68, 383-389.

17. McClements, D. J. and Decker, E. A. (2000) Lipid oxidation in oil-in-water emulsions: Impact of molecular environment on chemical reactions in heterogeneous food systems. $J$. Food Sci. 65, 1270-1282.

18. Muguerza, E., Ansorena, D., and Astiasarán, I. (2004) Functional dry fermented sausages manufactured with high levels of $n-3$ fatty acids: nutritional benefits and evaluation of oxi- dation. J. Sci. Food Agri. 84, 1061-1068.

19. Panpipat, W. and Yongsawatdigul, J. (2008) Stability of potassium iodide and omega-3 fatty acids in fortified freshwater fish emulsion sausage. LWT - Food Sci. Tech. 41, 483492.

20. Razminowicz, R. H., Kreuzer, M., and Scheeder, M. R. L. (2006) Quality of retail beef from two grass-based production systems in comparison with conventional beef. Meat Sci. 73, 351-361.

21. Serrano, A., Cofrades, S., and Jiménez-Colmenero, F. (2006) Characteristics of restructured beef steak with different proportions of walnut during frozen storage. Meat Sci. 72, 108115.

22. Shahidi, F. and Miraliakbari, H. (2004) Omega-3 (n-3) fatty acids in health and disease: Part 1--cardiovascular disease and cancer. J. Med. Food. 7, 387-401.

23. Uauy, R. and Valenzuela, A. (2000) Marine oils: the health benefits of n-3 fatty acids. Nutrition 16, 680-684.

24. Valencia, I., Ansorena, D., and Astiasarán, I. (2006) Nutritional and sensory properties of dry fermented sausages enriched with n-3 PUFAs. Meat Sci. 72, 727-733.

25. Zhang, S. X., Farouk, M. M., Young, O. A., Wieliczko, K. J., and Podmore, C. (2005) Functional stability of frozen normal and high pH beef. Meat Sci. 69, 765-772.

26. Ziegler, G. R., Rizvi, S. S. H., and Acton, J. C. (1987) Relationship of water content to textural characteristics, water activity, and thermal conductivity of some commercial sausages. J. Food Sci. 52, 901-905.

(Received 2014.9.27/Accepted 2014.12.3) 